\title{
Long-term Outcomes of Sacral Nerve Stimulation in Pelvic Floor Dysfunctions
}

\author{
David Hernández-Hernández ${ }^{1}$, Bárbara Padilla-Fernández ${ }^{1,2}$, Milagros Castro Romera ${ }^{3}$, Stephany Hess Medler \\ David Castro-Díaz ${ }^{1,2}$ \\ ${ }^{1}$ Servicio de Urología, Hospital Universitario de Canarias, Tenerife, Spain \\ ${ }^{2}$ Departamento de Cirugía, Facultad de Medicina, Universidad de La Laguna, Tenerife, Spain \\ ${ }^{3}$ Servicio de Cirugía, Hospital Universitario de Canarias, Tenerife, Spain \\ ${ }^{4}$ Departamento de Psicología Clínica, Psicobiología y Metodología, Universidad de La Laguna, Tenerife, Spain
}

\begin{abstract}
Purpose: The aim of this study was to analyze the long-term outcomes of sacral nerve stimulation (SNS) in both idiopathic and neurogenic pelvic floor disorders in patients treated at a referral center.

Methods: This retrospective observational study analyzed the records of 106 patients tested at our department from December 1999 to January 2017. The efficacy variables evaluated were the Global Response Assessment (range, 0\%-100\%) and, according to the clinical indication, other specific variables such International Consultation on Incontinence QuestionnaireShort Form, number of catheterizations or pads/day, and the numerical pain scale. The safety variables analyzed were complications (pain, migration, infection), reinterventions and explants. Patients' quality of life (QoL) and satisfaction with the procedure were evaluated through telephone interviews.

Results: The clinical indications were overactive bladder $(\mathrm{OAB})(\mathrm{n}=36)$, urinary retention $(\mathrm{UR})(\mathrm{n}=37)$, bladder pain syndrome/interstitial cystitis (BPS/IC) $(n=19)$, fecal incontinence (FI) $(n=8)$, and double incontinence (DI) $(n=6)$. The implant rates according to the clinical indication were as follows: OAB, 55.6\%; UR, 56.8\%; BPS/IC, 63.15\%; FI, 87.5\%; and DI, 66.7\%. Clinical and/or statistically significant improvements in all efficacy variables were observed. Loss of therapeutic effect at 75 months of follow-up was observed in 34\% of patients. Device-related pain appeared in 25 patients (39\%); in 20 patients, it was resolved by reprogramming and 5 patients required device removal. An overall improvement in QoL and high levels of satisfaction with the procedure were observed. More than $90 \%$ of patients would recommend SNS to a friend or relative. Conclusions: SNS is a minimally invasive procedure that offers a real alternative to patients with refractory pelvic floor dysfunction. Its safety profile is very favorable and it provides a long-lasting improvement in symptoms and QoL.
\end{abstract}

Keywords: Sacral neuromodulation; Efficacy; Bladder dysfunction; Fecal incontinence; Bladder pain syndrome

- Research Ethics: This study was approved by the local Ethics and Research Committee.

- Conflict of Interest: No potential conflict of interest relevant to this article was reported.

\section{INTRODUCTION}

Sacral nerve stimulation (SNS) is a well-established treatment for patients with chronic bowel or voiding dysfunction who do not respond to first- or second-line treatments (dietary and lifestyle modifications, pharmacotherapy, and pelvic floor muscle training) [1]. This heterogeneous group of pathologies includes overactive bladder $(\mathrm{OAB})$ with or without urinary inconti-

Corresponding author: David Hernández-Hernández

(iD https://orcid.org/0000-0003-3516-7620

Servicio de Urología, Hospital Universitario de Canarias, Carretera La Cuesta-

Taco, s/n 38320-La Laguna, Tenerife, Canary Islands, Spain

E-mail: david_hdezhdez@msn.com

Submitted: October 3, 2020 / Accepted after revision: November 23, 2020 
nence, chronic nonobstructive urinary retention (UR), and fecal incontinence (FI). SNS has also emerged as a therapeutic option in other indications, for which it can be used as a minimally invasive last resort before considering major surgery, such as augmentation cystoplasty or simple cystectomy (in cases of refractory chronic bladder pain syndrome/interstitial cystitis [BPS/IC]) [2] or colostomy (in the case of chronic persistent constipation) [3].

Its precise mechanisms of action are not fully understood, but SNS seems to promote normal bladder function by modulating spinal cord reflexes and brain networks $[4,5]$. The effect of SNS on pain disorders could be explained by the inhibition of interneurons located in the dorsal horn of the spinal cord, modulating pain sensation at higher brain centers (according to the gate control theory of pain proposed by Melzack and Wall) $[6,7]$.

Our hospital is a tertiary referral center for a population of 2.1 million inhabitants. This article aimed to summarize our experience with SNS from our first implant in December 1999 to January 2017, in order to provide mid- and long-term followup data regarding efficacy and complications.

\section{MATERIALS AND METHODS}

After receiving approval from the local Ethics and Research Committee, the medical records of all patients tested for SNS at our institution between December 1999 and January 2017 with at least 1 year of follow-up were reviewed. The inclusion criteria were as follows: patients aged 18 years or over with a signed informed consent form who were diagnosed with $\mathrm{OAB}$ (urgencyfrequency syndrome with or without urgency urinary incontinence), chronic nonobstructive UR, BPS/IC, FI, or double incontinence (urinary incontinence and FI), with at least 1 year of follow-up to ensure an adequate evaluation of mid- and longterm efficacy and safety variables. The exclusion criteria were underage and cognitively impaired patients.

Every patient was evaluated following our regular clinical practice (physical examination, imaging studies, urodynamics, and cystoscopy as indicated), and none of them responded to previous conservative treatment, which constituted behavioral therapy and pelvic floor muscle training, as well as antimuscarinics (and more recently $\beta 3$-agonists) in $\mathrm{OAB}$; oral agents and intravesical instillations in BPS/IC; and constipating agents and biofeedback in FI.

Sacral root (S3) acute stimulation was performed under local anesthesia in the outpatient clinic until 2003 (percutaneous nerve evaluation). Later, after the introduction of the Tined lead electrode, acute stimulation was carried out in the operating room under fluoroscopic guidance, as described by Spinelli et al. [8]. In case of positive sensory and/or motor response, the patient was sent home with an external pulse generator for the evaluation period. Then, 7-10 days later, the patient was evaluated with a clinical interview and questionnaires and asked about the symptoms. A positive response was defined as a subjective symptom improvement greater than or equal to $50 \%$ in the Global Response Assessment (GRA) (0\% "no response" to $100 \%$ "complete resolution of symptoms"), International Consultation on Incontinence Questionnaire-Short Form (ICIQ$\mathrm{SF})$ questionnaire, number of catheterizations per day, number of pads per day, and/or numerical pain score (0 to 10) in patients who experienced pain. In patients with a positive response that did not reach $50 \%$, we proceeded to device reprogramming and extended the evaluation period for 1 more week up to a maximum of 1 month. Patients showing a positive response received an implantable pulse generator (IPG). All surgeries were performed or supervised by the same senior surgeon. The follow-up schedule included a medical consultation every 6 months and, in case of changes in stimulation or the therapeutic effect, the device was checked and reprogrammed by the company technician. Clinically stable patients could be followed yearly.

For the assessment of quality of life (QoL) and satisfaction with the SNS procedure, a telephone interview was carried out by a nurse outside the urology unit, with 3 simple questions:

- Health-related QoL before and after SNS therapy. This QoL scale was adapted from the Spanish version of the EuroQol5D questionnaire [9] and scored health-related QoL from 0 ("the worst health you can imagine") to 100 ("the best health you can imagine")

- Satisfaction with the SNS procedure, with 0 being "extremely dissatisfied" and 10 "extremely satisfied"

- Would you recommend the SNS procedure to a friend or relative?

The data were collected and analyzed with IBM SPSS Statistics ver. 26.0 (IBM Co., Armonk, NY, USA). Categorical variables were described as frequencies and percentages. Quantitative variables were presented as mean and standard deviation or as median and percentiles, as appropriate. Survival analysis and the chi-square test were also performed.

The results were considered statistically significant at $\mathrm{P}<0.05$. 
Table 1. Patient distribution according to diagnosis and the success rate of sacral nerve stimulation (positive response to acute and subacute stimulation proceeding to IPG implant)

\begin{tabular}{lccc}
\hline Clinical diagnosis & $\begin{array}{c}\text { Patients undergoing acute nerve } \\
\text { evaluation }\end{array}$ & $\begin{array}{c}\text { Patients undergoing subacute } \\
\text { stimulation }\end{array}$ & $\begin{array}{c}\text { Patient receiving IPG } \\
\text { (successful subacute stimulation) }\end{array}$ \\
\hline OAB & $36(34)$ & $32(88.9)$ & $20(55.6)$ \\
UR & $37(34.9)$ & $30(81.1)$ & $21(56.8)$ \\
BPS/IC & $19(17.9)$ & $17(89.4)$ & $12(63.15)$ \\
FI & $8(7.5)$ & $7(87.5)$ & $7(87.5)$ \\
DI & $6(5.7)$ & $5(83.3)$ & $4(66.7)$ \\
Total & $106(100)$ & $91(85.9)$ & $64(60.4)$
\end{tabular}

Values are presented as number (\%).

IPG, implantable pulse generator; OAB, overactive bladder; UR, urinary retention; BPS/IC, bladder pain syndrome/interstitial cystitis; FI, fecal incontinence; DI, double incontinence.

\section{RESULTS}

During the study period, 106 tests were performed on 81 women (76.4\%) and 25 men (23.6\%). Patients' mean age at the time of the test was $52.9 \pm 13.5$ years (range, $18-78$ years). The clinical indications for SNS therapy and success rates are described in Table 1. The mean follow-up in our series was $75.35 \pm 55.10$ months, with a minimum of 14 months and a maximum of 220 months. Five implanted patients died of SNS-unrelated causes during the study period, and all of them had at least 1 year of follow-up.

Overall, 64 patients (60.4\%) were successfully tested and had a positive response during the evaluation period, and therefore we proceeded to an IPG implant. Three patients implanted in the group of BPS/IC were lost to follow-up. Nine patients in the $\mathrm{OAB}$ group and 14 patients in the UR group had underlying neurological conditions. Within these subgroups, the specific success rates were as follows: idiopathic and neurogenic $\mathrm{OAB}$, 55.6\%; idiopathic UR, 69.6\%; neurogenic UR, 35.7\%. The data on efficacy, survival analysis, complications, and reinterventions refer only to IPG-implanted patients.

\section{Efficacy}

In the OAB group, $77.8 \%$ of patients showed a GRA between $50 \%$ and $75 \%$, with statistically significant reductions in ICIQSF questionnaire scores (mean $15.69 \pm 4.79$ pre-SNS and $2.69 \pm$ 3.01 post-SNS) and pad use (mean $3.06 \pm 2.12$ pre-SNS and $0.88 \pm 1.26$ post-SNS). No differences were observed between idiopathic and neurogenic $\mathrm{OAB}$.

In the UR group, $40 \%$ of patients had spontaneous micturition without the need for catheterization. The mean number of catheterizations per day went from $4.45 \pm 1.98$ pre-SNS to $1.97 \pm 2.40$ post-SNS. In SNS responders, no differences were observed between idiopathic and neurogenic UR.

In the BPS/IC group, $83.4 \%$ of patients showed a GRA between $50 \%$ and $75 \%$, with subjective complete resolution of symptoms in $16.6 \%$ of patients. There was a statistically significant reduction in the numerical pain score from an average of $8.38 \pm 0.87$ points before SNS to $2.54 \pm 1.98$ points after SNS therapy $\left(-5.85\right.$ points, $\left.\mathrm{t}_{12}=9.45, \mathrm{P}<0.001\right)$.

In the FI group, $71.4 \%$ of patients reported subjective improvement in symptoms between $50 \%$ and $75 \%$, with $14.3 \%$ reporting complete resolution of symptoms. Pad use also decreased significantly from $4.00 \pm 2.10$ to $1.33 \pm 1.86$ postoperatively $\left(\mathrm{t}_{5}=12.65, \mathrm{P}<0.001\right)$.

Patients with double incontinence (urinary incontinence and FI) also showed significant reductions in average pad use (mean $5 \pm 2.71$ pre-SNS and $1.71 \pm 0.76$ post-SNS) and ICIQ-SF scores (mean $15.50 \pm 2.12$ pre-SNS and $1.50 \pm 2.21$ post-SNS).

\section{Survival Analysis}

A retrospective analysis of the therapy effect survival data was performed through a Kaplan-Meier survival analysis. Our results showed that in the first 24 months, $20 \%$ of patients reported a loss of effect. However, more than $60 \%$ of them benefited from SNS therapy for up to 14 years (Fig. 1).

$\mathrm{OAB}$ patients tended to have worse long-term outcomes, with a higher probability of loss of the therapeutic effect (Fig. 2). No significant differences were observed between patients with idiopathic and neurogenic lower urinary tract dysfunction (NLUTD) $\left(\chi^{2}=0.79, \mathrm{P}=0.375\right)$. 


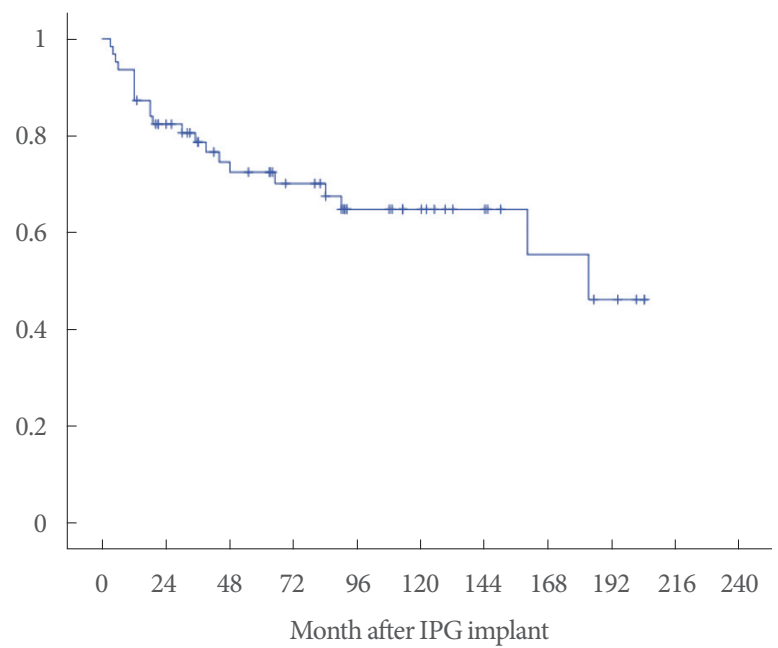

Fig. 1. Kaplan-Meier graph of the probability of sacral nerve stimulation effect survival. IPG, implantable pulse generator.

\section{Complications, Reinterventions, and Explants}

Complications were reported in $40.63 \%$ of patients (device-related pain, migration of the electrode or IPG, and infection). Twenty-five patients (39.06\%) reported device-related pain which was resolved in most cases by modifying the stimulation parameters. Only 5 patients required device explantation, all of them associated with a loss of effect of SNS.

One lead broke during explantation, requiring reintervention for the extraction of a small fragment, and one IPG migrated after a pelvic trauma; the device was extracted and relocated successfully. During the evaluation period, 1 patient suffered a local infection that required lead explantation. Overall, $12.5 \%$ of patients suffered complications requiring surgical intervention, while $31.25 \%$ had complications that were managed conservatively.

Slightly more than half of patients (51.6\%) required some type of reintervention, mostly constituting battery replacements (75\%). In one patient with neurogenic UR and partial response (from 6-7 catheterizations per day to 2-3 catheterizations per day) a second $\mathrm{S} 3$ electrode was tested without additional effect. In 5 cases, the battery replacement was combined with a new electrode owing to its failure (high impedance). In addition, complete device removal was carried out in 1 patient with UR and complete response due to the diagnosis of a brain tumor requiring multiple magnetic resonance imaging (MRI) studies. Patients affected by BPS/IC showed higher reoperation rates than expected, but this trend did not reach statistical significance $\left(\chi^{2}=6.22, \mathrm{P}=0.183\right)$.

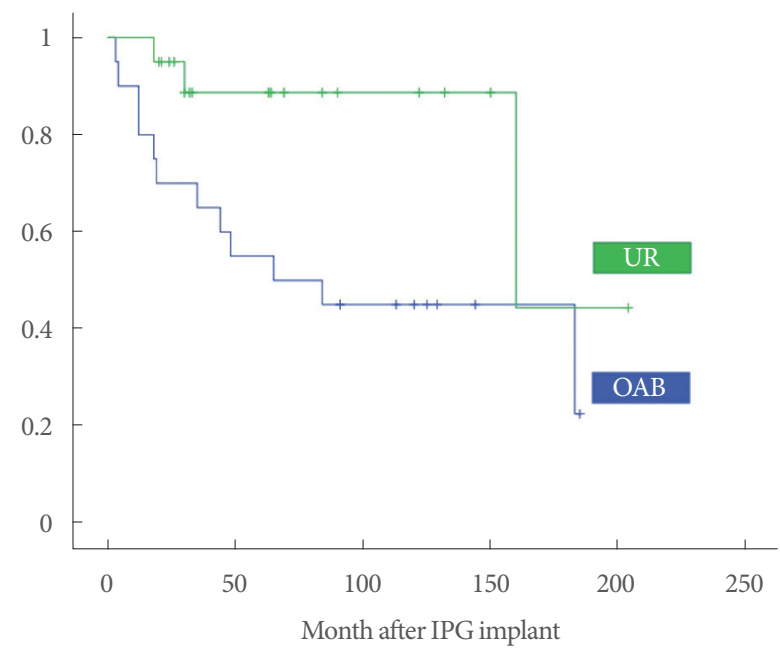

Fig. 2. Kaplan-Meier graph of the probability of sacral nerve stimulation effect survival in urinary retention (UR) and overactive bladder (OAB). IPG, implantable pulse generator.

The overall explantation rate was 9.4\% (6 of 64 devices). Five cases were due to pain associated with loss of effect, and the sixth, as mentioned above, was due to the need to perform repeated MRI studies.

\section{QoL and Satisfaction With the Procedure}

Phone interviews were performed by a nurse from a department uninvolved in the SNS procedure. A maximum of 3 phone calls to contact each patient were made, and $62.7 \%$ of patients were available to participate in the phone interview about their QoL and satisfaction with the procedure. According to patients' responses, we found significant differences between the pre-SNS (QoL-Pre) and post-SNS (QoL-Post) health scores $\left(\mathrm{F}_{1,32}=50.39, \mathrm{P}<0.001, \eta \mathrm{p}^{2}=0.612\right)$, with an overall improvement of 40.81 points (Table 2). In addition, $91.7 \%$ of patients stated that they would recommend SNS to a friend or relative.

\section{DISCUSSION}

Sixty-four out of 106 tested patients showed a favorable response to acute and subacute stimulation, proceeding to definitive implantation (60.4\% overall implant rate). These results are in line with other series, which are summarized in Table 3 [10-17].

Regarding patients with NLUTD, 9 of 36 patients in the OAB group (25\%) and 14 of 37 (37.8\%) in the UR group had underlying neurologic conditions, as follows: incomplete traumatic spinal cord injury (7 cases), multiple sclerosis (6 cases), Parkinson disease ( 2 cases), transverse myelitis ( 2 cases), lumbar spi- 
Table 2. Quality of life before SNS (QoL-Pre) and after SNS (QoL-Post)

\begin{tabular}{lccr}
\hline Clinical diagnosis & QoL-Pre & QoL-Post & P-value \\
\hline Overactive bladder & $52.73 \pm 16.94$ & $66.82 \pm 23.27$ & 0.0141 \\
Urinary retention & $30.38 \pm 20.15$ & $79.62 \pm 19.94$ & $<0.0001$ \\
BPS/IC & $17.86 \pm 16.04$ & $75.71 \pm 24.90$ & $<0.0001$ \\
Fecal incontinence & $5.00 \pm 10.00$ & $55.00 \pm 42.03$ & 0.003 \\
Double incontinence & $15.00 \pm 21.21$ & $70.00 \pm 28.28$ & 0.017 \\
Total & $31.08 \pm 23.37$ & $71.89 \pm 24.9$ & $<0.001$ \\
\hline
\end{tabular}

Values are presented as mean \pm standard deviation.

SNS, sacral nerve stimulation; QoL, quality of life; BPS/IC, bladder pain syndrome/interstitial cystitis.

Table 3. Summary of case series including response rates to SNS therapy

\begin{tabular}{|c|c|c|c|c|}
\hline Study & No. & Indication & Response rate & Follow-up (mo) \\
\hline Matzel et al. (2004) [10] & 34 & FI & $87 \%$ & 24 \\
\hline \multirow[t]{2}{*}{ White et al. (2008) [11] } & \multirow[t]{2}{*}{40} & \multirow[t]{2}{*}{ UR } & 74\% idiopathic UR & \multirow[t]{2}{*}{40} \\
\hline & & & $55 \%$ neurogenic UR & \\
\hline \multirow[t]{2}{*}{ Marcelissen et al. (2010) [12] } & \multirow[t]{2}{*}{64} & OAB, UR & $64 \%$ & \multirow[t]{2}{*}{53} \\
\hline & & UR & & \\
\hline \multirow[t]{3}{*}{ Al-Zahrani et al. (2011) [13] } & \multirow[t]{3}{*}{96} & $\mathrm{OAB}$ & $54.40 \%$ & \multirow[t]{3}{*}{51} \\
\hline & & UR & $43.90 \%$ & \\
\hline & & BPS/IC & $66 \%$ & \\
\hline Faucheron et al. (2012) [14] & 41 & DI & $87 \%$ & 62 \\
\hline \multirow[t]{2}{*}{ Peeters et al. (2014) [15] } & \multirow[t]{2}{*}{217} & $\mathrm{OAB}$ & \multirow[t]{2}{*}{$57 \%$} & \multirow[t]{2}{*}{47} \\
\hline & & UR & & \\
\hline Siegel et al. (2018) [16] & 272 & $\mathrm{OAB}$ & $67 \%$ & 60 \\
\hline \multirow[t]{4}{*}{ Zhang et al. (2019) [17] } & \multirow[t]{4}{*}{247} & $\mathrm{OAB}$ & $42.50 \%$ & \multirow[t]{4}{*}{20} \\
\hline & & UR & $51.60 \%$ & \\
\hline & & BPS/IC & $72.40 \%$ & \\
\hline & & NLUTD & $58.80 \%$ & \\
\hline \multirow[t]{5}{*}{ Present study } & \multirow[t]{5}{*}{64} & $\mathrm{OAB}$ & $55.60 \%$ & \multirow[t]{5}{*}{75} \\
\hline & & UR & $56.80 \%$ & \\
\hline & & BPS/IC & $63.15 \%$ & \\
\hline & & $\mathrm{FI}$ & $87.50 \%$ & \\
\hline & & DI & $66.70 \%$ & \\
\hline
\end{tabular}

SNS, sacral nerve stimulation; FI, fecal incontinence; UR, urinary retention; OAB, overactive bladder; BPS/IC, bladder pain syndrome/interstitial cystitis; NLUTD, neurogenic lower urinary tract dysfunction; DI, double incontinence.

nal stenosis ( 2 cases), myelomeningocele ( 1 case), cauda equina syndrome (1 case), anal atresia (1 case), and encephalitis associated with systemic lupus erythematosus (1 case).

In our series, patients with NLUTD had a lower response rate than their idiopathic counterparts ( $47.82 \%$ vs. $66.26 \%)$. The response to SNS in patients with idiopathic and neurogenic $\mathrm{OAB}$ was the same (55.6\% of positive responses in patients who underwent IPG implantation). In contrast, neurogenic UR showed a significantly lower positive response rate than idiopathic UR (35.7\% vs. $69.6 \%$, respectively). These results are in accordance with those published in a systematic review and meta-analysis by Kessler et al. [18] in 2010, bringing together 34 studies with 
implant rates for patients with NLUTD between 29\% and $100 \%$. White published similar results, with implant rates of $74 \%$ in patients with idiopathic UR and 55\% in those with neurogenic UR [11].

Although the efficacy and safety of SNS are well established in the short- and mid-term, long-term data (more than 60 months) are scarce. As observed in our series, there was a loss of effect particularly within the first 2 years, with SNS becoming ineffective in $20 \%$ of patients. This trend improved, and the probability of maintaining favorable therapeutic effects beyond 10 years was greater than $60 \%$. This finding has also been reported by other series with medium- and long-term follow-up. Peeters et al. [15] in a retrospective analysis of 217 patients implanted between 1996 and 2010 with a mean follow-up of 47 months, reported a loss of effect in $29 \%$ of patients. A study by Ismail et al. [19], including $34 \mathrm{OAB}$ patients with a mean follow-up of 9.7 years, showed a loss of effect of therapy in $47 \%$. These results are similar to our series, since we observed that the loss of effect of therapy was more pronounced in patients with $\mathrm{OAB}$, with $50 \%$ of implanted patients no longer benefiting from therapy at 6 years of follow-up. However, UR patients showed a more sustained clinical response, with more than $85 \%$ of patients showing a positive effect of SNS therapy beyond 6 years. In agreement with our observations, Elhilali et al. [20], in a retrospective analysis of 52 patients (28 with OAB, 9 with UR, and 4 with BPS/IC), reported that $78 \%$ of patients with UR had a sustained positive effect compared to $52 \%$ of patients with $\mathrm{OAB}$, with a mean follow-up of 77 months.

The current guidelines of the European Association of Urology and American Association of Urology recommend SNS for BPS/IC patients in their treatment algorithms before considering invasive surgical treatment [21,22]. The evidence for this recommendation is based mainly on small retrospective case series with heterogeneous patient populations and limited follow-up; consequently, SNS has not been approved by the Food and Drug Administration for this indication. However, the use of SNS in BPS/IC is increasingly common since the early reports in 1999 by Shaker and Hassouna [23]. A recent systematic review and meta-analysis published by Wang et al. [24] included more than 500 patients with a follow-up between 0 (results of SNS test) to 86 months. The primary outcomes were reduction of pain, reduction of scores on the Interstitial Cystitis Symptoms Index (ICSI) and Interstitial Cystitis Problem Index (ICPI) questionnaires, and the success rate. The average reduction in pain was 3.99 points on a $0-10$ visual analogue scale.
The ICSI/ICPI questionnaire scores were also significantly lower after SNS therapy, and the success rates ranged from $60 \%$ to $98 \%$. The complication rate ranged between $0 \%$ and $56 \%$, with an explantation rate of $8 \%$. The short-term results were similar to the medium- and long-term results ( $88 \%$ success vs. $76 \%$ success), not confirming previously reported findings suggesting that the effect of SNS therapy could be shorter-lasting in BPS/IC than in other indications [13]. Our results, with a mean follow-up of 96 months, agree with this meta-analysis regarding the reduction of pain, complications, and the duration of SNS therapy effect.

Matzel et al. [25] published the first series of SNS for the treatment of patients with FI and intact sphincters in 1995. After this report, SNS spread widely in the management of FI, even with sphincter muscle defects, with multiple series demonstrating positive results. In 2011, a meta-analysis by Tan et al. [26] with 944 patients reported a response rate of $70.4 \%$. Significant improvements in QoL and the Wexner incontinence scale, as well as a decrease in the number of weekly incontinence episodes, were demonstrated, which were independent of the degree of sphincter injury. These results were accompanied by a complication rate of $15 \%$ and an explantation rate of $3 \%$. We observed slightly higher response rates in our series (87\%) and, with a mean follow-up of 61.4 months, a loss of effect in $28.5 \%$ of patients. Similar findings have also been reported in a retrospective series with 325 patients implanted with a mean followup of 85 months (32\% loss of effect) [27].

Regarding patients affected simultaneously by urinary incontinence and FI, 4 of 6 responded successfully to the test stages and proceeded to an IPG implant. In 1 case, there was partial improvement of both types of incontinence, and another case experienced complete resolution of urinary incontinence but persistence of FI, which finally required colostomy. We observed a complete response, with urinary and fecal continence, in 2 patients, of whom 1 experienced symptom recurrence after 6 months. The results on double incontinence published so far are scarce and quite heterogeneous, with case series containing between 3 and 57 patients and a mean maximum follow-up of 62 months [28]. However, after the failure of pharmacotherapy and physical therapy/biofeedback, SNS is the only surgical treatment available for double incontinence. In the review published by Chodez et al. [28] in 2014, the response rates varied from $44 \%$ to $100 \%$ for FI and from $20 \%$ to $100 \%$ for urinary incontinence, with approximately $50 \%$ of patients showing improvement in both urinary and fecal symptoms. Despite this, 
the series had a limited number of patients and the longest follow-up did not exceed 62 months ( 23 months on average).

The limitations of our study include a small sample size, especially for patients with FI and double incontinence. Furthermore, the retrospective nature of the study prevented us from collecting bladder diary data in all patients, meaning that these were not available for further analysis. Additionally, the use of phone interviews to evaluate satisfaction could favor survey and respondent bias.

In conclusion, SNS is an effective and safe technique in a complex group of patients with voiding and/or intestinal dysfunction or chronic bladder pain, refractory to conservative measures and pharmacological treatments. Its safety profile is very favorable, with mild complications. Moderate reintervention rates and a loss of effect must be expected in the long term. Nonetheless, SNS noticeably improves QoL in responders to therapy.

\section{AUTHOR CONTRIBUTION STATEMENT}

- Conceptualization: $D H H, B P F, D C D$

- Data curation: $D H H, M C R$

- Formal analysis: $S H M$

- Methodology: $D H H, B P F, S H M, D C D$

- Project administration: $D H H, D C D$

- Writing-original draft: $D H H$

-Writing-review \& editing: $D H H, B P F, D C D$

\section{ORCID}

David Hernández-Hernández

0000-0003-3516-7620

Bárbara Padilla-Fernández

Stephany Hess Medler

David Castro-Díaz

\section{REFERENCES}

1. Oerlemans DJ, van Kerrebroeck PE. Sacral nerve stimulation for neuromodulation of the lower urinary tract. Neurourol Urodyn 2008;27:28-33.

2. Fariello JY, Whitmore K. Sacral neuromodulation stimulation for IC/PBS, chronic pelvic pain, and sexual dysfunction. Int Urogynecol J 2010;21:1553-8.

3. Altomare DF, Ratto C, Ganio E, Lolli P, Masin A, Villani RD. Longterm outcome of sacral nerve stimulation for fecal incontinence.
Dis Colon Rectum 2009;52:11-7.

4. Abello A, Das AK. Electrical neuromodulation in the management of lower urinary tract dysfunction: evidence, experience and future prospects. Ther Adv Urol 2018;10:165-73.

5. Kessler TM, de Wachter S. Neuromodulation of lower urinary tract dysfunction. Der Urologe Ausg A 2017;56:1591-6. (German).

6. Melzack R, Wall PD. Pain mechanisms: a new theory. Science 1965; 150:971-9.

7. Tahseen S. Role of sacral neuromodulation in modern urogynaecology practice: a review of recent literature. Int Urogynecol J 2018; 29:1081-91.

8. Spinelli M, Giardiello G, Arduini A, van den Hombergh U. New percutaneous technique of sacral nerve stimulation has high initial success rate: preliminary results. Eur Urol 2003;43:70-4.

9. Badia Llach X, Herdman M, Schiaffino A. Determining correspondence between scores on the EQ-5D "thermometer" and a 5-point categorical rating scale. Med Care 1999;37:671-7.

10. Matzel KE, Kamm MA, Stösser M, Baeten CG, Christiansen J, Madoff R, et al. Sacral spinal nerve stimulation for faecal incontinence: multicentre study. Lancet 2004;363:1270-6.

11. White WM, Dobmeyer-Dittrich C, Klein FA, Wallace LS. Sacral nerve stimulation for treatment of refractory urinary retention: long-term efficacy and durability. Urology 2008;71:71-4.

12. Marcelissen TA, Leong RK, de Bie RA, van Kerrebroeck PE, de Wachter SG. Long-term results of sacral neuromodulation with the tined lead procedure. J Urol 2010;184:1997-2000.

13. Al-zahrani AA, Elzayat EA, Gajewski JB. Long-term outcome and surgical interventions after sacral neuromodulation implant for lower urinary tract symptoms: 14-year experience at 1 center. J Urol 2011;185:981-6.

14. Faucheron JL, Chodez M, Boillot B. Neuromodulation for fecal and urinary incontinence: functional results in 57 consecutive patients from a single institution. Dis Colon Rectum 2012;55:127883.

15. Peeters K, Sahai A, De Ridder D, Van Der Aa F. Long-term followup of sacral neuromodulation for lower urinary tract dysfunction. BJU Int 2014;113:789-94.

16. Siegel S, Noblett K, Mangel J, Bennett J, Griebling TL, Sutherland $\mathrm{SE}$, et al. Five-year followup results of a prospective, multicenter study of patients with overactive bladder treated with sacral neuromodulation. J Urol 2018;199:229-36.

17. Zhang P, Wang JY, Zhang Y, Liao L, Lv JW, Ling Q, et al. Results of sacral neuromodulation therapy for urinary voiding dysfunction: five-year experience of a retrospective, multicenter study in China. Neuromodulation 2019;22:730-7. 
18. Kessler TM, La Framboise D, Trelle S, Fowler CJ, Kiss G, Pannek J, et al. Sacral neuromodulation for neurogenic lower urinary tract dysfunction: systematic review and meta-analysis. Eur Urol 2010; 58:865-74.

19. Ismail S, Chartier-Kastler E, Perrouin-Verbe MA, Rose-Dite-Modestine J, Denys P, Phé V. Long-term functional outcomes of s3 sacral neuromodulation for the treatment of idiopathic overactive bladder. Neuromodulation 2017;20:825-9.

20. Elhilali MM, Khaled SM, Kashiwabara T, Elzayat E, Corcos J. Sacral neuromodulation: long-term experience of one center. Urology 2005; 65:1114-7.

21. Engeler D, Baranowski AP, Berghmans B, Borovicka J, Cottrell AM, Dinis-Oliveira $\mathrm{P}$, et al. EAU guidelines on chronic pelvic pain. Arnhem (The Netherlands): EAU Guidelines Office; 2020.

22. Hanno PM, Erickson D, Moldwin R, Faraday MM; American Urological Association. Diagnosis and treatment of interstitial cystitis/ bladder pain syndrome: AUA guideline amendment. J Urol 2015; 193:1545-53.

23. Shaker H, Hassouna MM. Sacral root neuromodulation in the treatment of various voiding and storage problems. Int Urogynecol J Pelvic Floor Dysfunct 1999;10:336-43.

24. Wang J, Chen Y, Chen J, Zhang G, Wu P. Sacral neuromodulation for refractory bladder pain syndrome/interstitial cystitis: a global systematic review and meta-analysis. Sci Rep 2017;7:11031.

25. Matzel KE, Stadelmaier U, Hohenfellner M, Gall FP. Permanent electrostimulation of sacral spinal nerves with an implantable neurostimulator in treatment of fecal incontinence. Chirurg 1995; 66:813-7. (German).

26. Tan E, Ngo NT, Darzi A, Shenouda M, Tekkis PP. Meta-analysis: sacral nerve stimulation versus conservative therapy in the treatment of faecal incontinence. Int J Colorectal Dis 2011;26:275-94.

27. Janssen PT, Kuiper SZ, Stassen LP, Bouvy ND, Breukink SO, Melenhorst J. Fecal incontinence treated by sacral neuromodulation: Long-term follow-up of 325 patients. Surgery 2017;161:1040-8.

28. Chodez M, Trilling B, Thuillier C, Boillot B, Barbois S, Faucheron JL. Results of sacral nerve neuromodulation for double incontinence in adults. Tech Coloproctol 2014;18:1147-51. 